\title{
Boundary layer variational principles: A case study
}

\author{
Miroslav Grmela \\ École Polytechnique de Montreal, H3C 3A7 Montréal (Québec), Canada \\ Iliya V. Karlin * \\ Swiss Federal Institute of Technology, Department of Materials, Institute of Polymers \\ ETH-Zentrum, Sonneggstr. 3, ML J 19, CH-8092 Zürich, Switzerland \\ Vladimir B. Zmievski \\ École Polytechnique de Montreal, H3C 3A7 Montréal (Québec), Canada
}

\begin{abstract}
Considering the model heat conduction problem in the setting of Grad's moment equations, we demonstrate a crossover in the structure of minima of the entropy production within the boundary layer. Based on this observation, we formulate and compare variation principles for solving the problem of boundary conditions in nonequilibrium thermodynamics.
\end{abstract}

05.70.Ln, 47.45.-n, 51.10.+y

Typeset using REVTEX

${ }^{*}$ Corresponding author. E-mail: ikarlin@ @ifp.mat.ethz.ch 


\section{INTRODUCTION}

The goal of this paper is to study possibilities of formulating variational principles for boundary conditions appearing in the extended thermodynamic systems, where the usual locally conserved fields (the mass density, the momentum density, and the energy density) are supplemented by various non-conserved fields such as extra stresses in rheology [1] , higher-order moments of the one-particle distribution function in moment systems derived in kinetic theory of gases and plasmas, and many others. In order to be specific, we shall restrict our attention to the case of so-called extended thermodynamic system, underpinned by Grad's moment method of the Boltzmann equation of rarefied gas [2]. Since the seminal work of Grad [2], it is well known that the stationary problems in moment equations is ill-posed. Indeed, on physical grounds, it is often unclear how to infer the values of the higher moments on the boundaries without a more microscopic considerations.

In a situation where imposing boundary conditions is problematic or ill-posed, two main directions in the search for formulations of the boundary conditions can be distinguished. The first direction can be broadly characterized as a variational approach. A typical and quite well known representative of this strategy are so-called natural variational formulations of stationary equations [3]. This approach is widely used, in particular, in numerical methods based on local minimization schemes, such as the finite elements method [4]. Without going into any detail here, we mention that if the solution can be written as a minimizer of a functional, then it is sometimes possible to extend the solution from the bulk to the boundary, or to modify the functional in such a way as to make this extension possible. By doing so, the natural variational formulations results in so-called natural boundary conditions. Many examples are given in the standard references on the finite elements method [ 4 ]. It should be also noticed that the physical significance of the boundary condition thus arising is rarely addressed, especially in the case of extra fields without direct physical interpretation. The physics that is behind the behavior in the bulk may not be identical with the physics that is behind the boundary conditions. For example, new type of forces arise often on boundaries. It is thus possible that a direct extension to boundaries of the potentials that are found 
to express the physics in the bulk is unrealistic.

The second strategy is based on an attempt to express the physics that takes place on the boundaries. Let us assume that as the result of the physical analysis one formulates a coupled system of equations governing the time evolution in both the bulk and on boundaries. States on the boundary, or in a boundary layer, are described by values on the boundary (or in boundary layers) of the fields chosen to describe states in the bulk and possibly by some other fields defined only on boundaries or boundary layers. Some of the boundary state variables are fixed by an outside influence. The rest of them, the uncontrollable boundary state variables, evolve in time together with the bulk state variables. Let us assume that analysis of the time evolution equations shows that the uncontrolled boundary state variables evolve faster than the bulk state variables and that they approach, as the time goes to infinity, stationary values. These asymptotically reached stationary values of the boundary state variables are then the boundary conditions that we look for. We obtained them thus by solving the time evolution equations. If in addition, we are able to recognize in the analysis of the fast time evolution a Lyapunov functional, then also this second strategy becomes a variational method. This is because the boundary conditions we look for are in such a case extremal values of the Lyapunov functional. It is important to emphasize that the way the variational functional is introduced in this second strategy does not use the potential arising in the bulk, it does not even use the assumption that such potential exists. In fact, it is well known [5] that the time evolution in the bulk of driven systems can not be often associated with any potential. The potential introduced in the second strategy arises from the time evolution of the boundary state variables and not from the time evolution in the bulk.

The second strategy has been mentioned in Ref. [6] as an illustration of a general approach to the thermodynamics of driven systems. The potential-driven time evolution of boundary state variables have also been used in [7] in the context of the investigation of consequence of the stickslip boundary conditions in flows of polymeric liquids. The authors of Ref. [7] do not discuss the physical derivation of the boundary time evolution. Also the potential is introduced in [7] completely phenomenologically.

Our study in this paper remains also on a phenomenological level. We do not discuss the 
explicitly the boundary time evolution, we are not therefore in position to recognize the pertinent potential in its analysis. We have to use different considerations in order to identify it. Below, we shall follow a recent work of Struchtrup and Weiss [8] (see also Ref. [9]). Struchtrup and Weiss [8] proceed in three steps.

First, they suggest to consider the local entropy production $\sigma$ as a candidate for the potential that will eventually determine the missing boundary conditions. While it is quite well known [5] that $\sigma$ cannot always be directly related to the time evolution in the bulk, it can still be relevant to the boundary conditions (especially in the light of our expectation - based on the physical analysis sketched in the previous paragraph - that the boundary time evolution can always be associated with a potential).

Second, having chosen $\sigma$, one has to ask the question how does this potential depend on the boundary conditions. Struchtrup and Weiss [8] answer this question as follows: First, they limit the analysis to stationary solutions. Let the stationary solution corresponding to a given boundary condition is found. The entropy production $\sigma$, evaluated on the stationary solution, becomes a function of both the bulk and the boundary state variables.

So far, we have arrived at a potential depending on the bulk and the boundary state variables. What remains is to make the third step, and eliminate the dependence on the bulk variables. It is this third step where our analysis differs from Refs. [8,97]. It has been noticed in Ref. [8] that elimination of the bulk variables by averaging the entropy production over the entire volume - and which eventually leads to the total entropy production principle in a spirit of Glansdorff and Prigogine [10,11] - gives apparently wrong results in applications to the boundary condition problem. Instead, a different, much more local analysis has been adopted in [8,9]. However, the physical significance of such modifications, as well as the physical reasons why the global averaging out the bulk variables is not be working have not been addressed.

In this paper we address the question how physically meaningful variational principles for boundary conditions can be constructed on the basis of the entropy production by exploring more possibilities than those explored in [8,9]. The intuitive idea behind our consideration is that the additional variables in stationary problems often have a significance of a description of the bound- 
ary layer (this description is greatly reduced, as compared to a full kinetic equation). By adopting this viewpoint, we study the question as to what happens if the entropy production is considered not in the total volume of the system but rather is localized to sufficiently thin boundary layers. A physical interpretation of our results is as follows: If the domain of integration of the entropy production is restricted to sufficiently thin boundary layers, the result of the type minimization suggests the optimal choice of the boundary condition. Moreover, shrinking the domain where the entropy production is sampled from the whole bulk to the boundary layer reveals a behavior typical for a critical phenomena, with the optimal value of the boundary condition appearing as a result of passing a critical size of the layer. Various features of this transition are studied, and plausible realizations of the minimum principle are suggested.

\section{ENTROPY PRODUCTION IN THE BOUNDARY LAYER}

In the context of Grad's method [2] and its variations, the state of the system is described by the locally conserved fields $M(\boldsymbol{x}, t)$ (the local density, momentum and energy), and a finite number of nonconserved fields, $N(\boldsymbol{x}, t)$ (nonequilibrium stress tensor, heat flux, fluxes thereof etc). The fields $N$ are usually higher order moments of the distribution function which gives a full description of the system at a more microscopic level of the kinetic equation. Grad's method reduces in a systematic way the description from the level of the kinetic equation for the one-body distribution function to the level of a closed set of the moment equations involving only the fields $M$ and $N$. The nonlinear coupled sets of equations in partial derivatives are generically referred

to as Grad's moment equations, and are given in many sources. The original Grad's method [2], technically based on a Hermite polynomial expansion of the one-body distribution function satisfying the Boltzmann kinetic equation, has been extended and modified by many authors for various kinetic equations [12-14]. In particular, a generalization of Grad's method to non-moment variables has been addressed in [15-17]. Examples of Grad's moment equations will be considered in the next section. Here we remind that, each Grad's moment system is equipped with the function of the fields, $\sigma$, the local entropy production. Function $\sigma$ is nonnegative and equals to zero only at 
the local equilibrium, and it can be computed once the dissipative terms in the underlying kinetic equation are specified (for example, once the Boltzmann collision integral is specified). The form of the entropy production also depends on the version of Grad's method used in the derivation of moment equations. In many applications, the typical outcome for the entropy production is a quadratic form in the fields $N$ (this is valid for small deviations from local equilibrium),

$$
\sigma=\sum_{i j}\left(N_{i}-N_{i}^{\mathrm{eq}}(M)\right) A_{i j}(M)\left(N_{j}-N_{j}^{\mathrm{eq}}(M)\right)
$$

where $N_{i}^{\mathrm{eq}}(M)$ are values of the nonconserved fields in the local equilibrium (in terms of the kinetic theory, the latter is given by the local equilibrium distribution function which depends perimetrically only on the locally conserved fields $M$, the standard example is the local Maxwell distribution function), and where $A_{i j}$ is the positive semidefinite matrix, with matrix elements dependent on the functions $M$ and also on the details of particle's interaction in the kinetic picture (scattering cross-sections, for example).

In order to solve the stationary version of Grad's moment equations for the time-independent fields, $M(\boldsymbol{x})$ and $N(\boldsymbol{x})$, in a domain $U \in R^{n}$, with the boundary $\partial U$, a set of boundary conditions should be provided. In the typical situation, which we here assume, the boundary conditions for the locally conserved fields $M(\boldsymbol{x})$ are known, and the question concerns only the additional fields $N(\boldsymbol{x})$. To this end, we adopt the first two steps as suggested by Struchtrup and Weiss [8]: First, we consider the set of all possible solutions to stationary Grad's equations with the fixed boundary conditions for the conserved fields, $M_{\mathrm{b}}=\left.M(\boldsymbol{x})\right|_{\partial U}$, and with various boundary conditions for the nonconserved fields, $N_{\mathrm{b}}=\left.N(\boldsymbol{x})\right|_{\partial U}$. (In principle, other types of boundary conditions could be addressed, including derivatives of either $M$ or $N$, but we shall not consider this option here). Second, evaluating the local entropy production functional (11) on the configurations of the fields thus obtained, we get a set of functions, $\sigma\left(\boldsymbol{x}, N_{\mathrm{b}}\right)$, parameterized by the boundary condition data $N_{\mathrm{b}}$. Finally, in the third step, one has to eliminate the dependence on $\boldsymbol{x}$, and to end up with a potential $\Psi\left(N_{\mathrm{b}}\right)$ depending only on the boundary data $N_{\mathrm{b}}$, and whose minima should suggest the choice of the boundary condition. It is this third step where we offer a more detailed analysis, as compared to Refs. [8,9]. 
Specifically, we introduce an additional structure into the domain $U$. Being inspired by the concept of the boundary layer, we introduce a one-parametric family of subdomains $B_{L}$, where $L \geq 0$. Each $B_{L}$ (the test boundary layer) is thought as a subdomain of the characteristic thickness $L$, attached to the boundary $\partial U$. For the test boundary layer $B_{L}$, we consider the layer-averaged entropy production,

$$
\Sigma_{B_{L}}\left(N_{\mathrm{b}}\right)=\frac{1}{\operatorname{Vol}\left(B_{L}\right)} \int_{B_{L}} \sigma\left(\boldsymbol{x}, N_{\mathrm{b}}\right) d \boldsymbol{x},
$$

where $\operatorname{Vol}\left(B_{L}\right)$ is the volume of the subdomain $B_{L}$.

The study of minimizers of the set of functionals (2) for various characteristic thicknesses $L$ used to define the boundary layer is the central point of our paper. A priori, it is clear that, if the thickness of the layer is taken large enough, then we eventually come to the total bulk-averaged entropy production,

$$
\Sigma_{U}\left(N_{\mathrm{b}}\right)=\frac{1}{\operatorname{Vol}(U)} \int_{U} \sigma\left(\boldsymbol{x}, N_{\mathrm{b}}\right) d \boldsymbol{x} .
$$

As it has been already demonstrated with explicit examples in Ref. [8], minimization of the functionals (3) over the boundary data $N_{\mathrm{b}}$ selects the field configurations beyond a reasonable physical interpretation. On the other hand, if we go into the opposite direction, taking thinner test boundary layers, and if the hypothesis about the fields $N$ as playing the most important role in the description of the physical boundary layer is right, we might expect a crossover in the structure of the minimizers of the functional (2). Specifically, we expect that at some value $L_{\mathrm{c}}$, a local minimum will start appearing, and which would correspond to the physically plausible value of the boundary condition $N_{\mathrm{b}}$. We further expect that variations of these minimal values is not large for the entire interval $L \in\left[0, L_{\mathrm{c}}\right]$.

This expectation is also motivated in part by the suggestion of Struchtrup and Weiss [8] who postulated a much more local functional as compared to the total bulk-averaged entropy production (3), namely, that correct configurations should minimize the maximum of the local entropy production, thus, considering the functional,

$$
\Psi_{\mathrm{sw}}\left(N_{\mathrm{b}}\right)=\max _{\boldsymbol{x} \in U} \sigma\left(\boldsymbol{x}, N_{\mathrm{b}}\right)
$$


Our suggestion to study functionals (2) which sample the entropy production more locally in space as compared to the total entropy production (3) does not coincide with the "ultralocal" functional (Ф), and results are not expected to be identical even in the one-dimensional cases considered below. It should be stressed that a correct mathematical definition of the system of the test boundary layers $B_{L}$ requires more restrictions but we here do not consider this point rigorously here. Finally, the notion of the boundary layer is pertinent to the underlying kinetic theory where it can be computed in a few model settings [18]. However, it is not straightforward to incorporate these results into our considerations.

Notice that the above construction does not eliminate completely the $\boldsymbol{x}$-dependence from the local entropy production $\sigma\left(\boldsymbol{x}, N_{\mathrm{b}}\right)$, rather, it replaces such a dependence by a more transparent oneparametric dependence on the thickness $L$. In principle, any functional $\Sigma_{B_{L}}$ for $L \in\left[0, L_{\mathrm{c}}\right]$ can be regarded as a potential. However, in practice, a priori estimates for the characteristic value of $L$ are sometimes available. These values can be dependent on boundary conditions for controllable and uncontrollable fields as well (see next section). On the other hand, the set of the subdomains suggests a realization for the potential $\Psi\left(N_{\mathrm{b}}\right)$ which compares the averaged entropy production within the layer $B_{L}$ with the averaged entropy production within the rest of the bulk, $U \backslash B_{L}$,

$$
\Sigma_{U \backslash B_{L}}\left(N_{\mathrm{b}}\right)=\frac{1}{\operatorname{Vol}\left(U \backslash B_{L}\right)} \int_{U \backslash B_{L}} \sigma\left(\boldsymbol{x}, N_{\mathrm{b}}\right) \mathrm{d} \boldsymbol{x} .
$$

Namely, smoothness of transition from the boundary layer into the bulk suggests the outcome for the boundary condition $N_{\mathrm{b}}$ which guarantees that the difference between the averaged entropy production in the boundary layer and the averaged entropy production in the bulk is minimal. This results in a minimization of the potential,

$$
\Psi_{1}\left(N_{b}\right)=\left|\Sigma_{B_{L}}\left(N_{\mathrm{b}}\right)-\Sigma_{U \backslash B_{L}}\left(N_{\mathrm{b}}\right)\right|
$$

Other variational principles can be constructed on similar grounds. In particular, if one expects that a variation of the entropy production in the boundary layer is considerably higher than in the bulk, then the functional $\Psi_{1}$ (16) can be replaced with a functional involving only local measures of activity 


$$
\Psi_{2}\left(N_{b}\right)=\max _{\boldsymbol{x} \in B_{L}} \sigma\left(\boldsymbol{x}, N_{\mathrm{b}}\right)-\min _{\boldsymbol{x} \in B_{L}} \sigma\left(\boldsymbol{x}, N_{\mathrm{b}}\right)
$$

Here $\min {\boldsymbol{x} \in B_{L}}_{\sigma} \sigma\left(\boldsymbol{x}, N_{\mathrm{b}}\right)$ represents an approximation to the bulk activity. In the next section we shall test all this in the context of a model of heat transfer.

\section{ONE DIMENSIONAL HEAT CONDUCTION PROBLEM}

Following Refs. [8,9], we consider here a one-dimensional problem of a stationary heat transfer for Boltzmann's gas at rest placed between two walls with fixed temperatures. The system is described by Grad's 14-moment equations. The set of field variables includes hydrodynamic fields $M$ (the mass density $\rho(\boldsymbol{x})$, the average velocity $\boldsymbol{v}(\boldsymbol{x})$, and the temperature $T(\boldsymbol{x})$ ), as well as the additional variables $N$, which are functions of higher moments: The stress tensor $\tau(\boldsymbol{x})$, the heat flux $\mathbf{q}(\boldsymbol{x})$, and one more scalar field, $\Delta(\boldsymbol{x})$, which corresponds to the fourth-order moment of the one-particle distribution function,

$$
\Delta(\boldsymbol{x})=\int_{R^{3}}\left[f(\boldsymbol{v}, \boldsymbol{x})-f^{\mathrm{eq}}(M(\boldsymbol{x}), \boldsymbol{v})\right] v^{4} d \boldsymbol{v}
$$

where $f^{\text {eq }}$ is the local Maxwellian.

We further assume that Grad's 14-moment distribution function, $f_{14}(M(\boldsymbol{x}), N(\boldsymbol{x}), \boldsymbol{v})$, depends only on one spatial variable $x$, and that the velocity dependence is symmetric with respect to rotations in the $\left(v_{y}, v_{z}\right)$ plane. In this case, the average velocity vector, the traceless part of the stress tensor $\stackrel{\circ}{\tau}$, and the $y$-, and the $z$-components of the heat flux vector, are equal to zero. The 14-moment Grad's system reduces to the system of four equations for the mass density $\rho(x)$, for the pressure $p(x)$, for the heat flux $q(x)$, and for the fourth moment, $\Delta(x)$, and it reads [8, 9, 13]:

$$
\begin{aligned}
\partial_{x} q & =0, \\
\partial_{x} p & =0, \\
\partial_{x}\left(\Delta+15 \frac{p^{2}}{\rho}\right) & =-6 \frac{1}{\xi_{1}(\rho, T)} q, \\
\partial_{x} \frac{q p}{\rho} & =-\frac{1}{28} \frac{1}{\xi_{2}(\rho, T)} \Delta .
\end{aligned}
$$


Here the pressure $p(\boldsymbol{x})$ is related to the temperature, and the density as $p=(k / m) \rho T$, and the positive coefficients $\xi_{1}$ and $\xi_{2}$ are the relaxation times, which can be functions of the density $\rho$ and the temperature $T$. Explicit form of parameters $\xi_{1,2}$ is determined by the collision model used in the corresponding Boltzmann equation. Following [8,9] we consider two models: the BhatnagarGross-Krook equation (BGK model) which gives constant relaxation times $\xi_{1}=\xi_{2}=\tau$, and the gas of Maxwell molecules (MM model) which leads to the choice $\xi_{1}=\xi_{2}=2 / 3(\alpha \rho)$, where $\alpha$ is a constant. The local entropy production for Grad's system (8) reads:

$$
\sigma=\frac{m}{k} \frac{1}{p T}\left\{\frac{2}{5} \frac{1}{\xi_{1}} \frac{1}{T} q^{2}+\frac{1}{120} \frac{1}{\xi_{2}} \frac{m}{k} \frac{\Delta^{2}}{T^{2}}\right\}
$$

We assume that the walls are placed at $x=0$ and $x=a$. Taking into account the fact that the pressure and the heat fluxes are constant, the equations (8) require one additional boundary condition (in addition to boundary conditions for the temperature $T(0)=T_{0}, T(a)=T_{1}$ ) in order to fix either the heat flux $q$ or the variable $\Delta$ at one of the boundaries.

Let us first consider the BGK model. It proves convenient to introduce reduced variables,

$$
\begin{aligned}
T^{\prime}=\frac{T}{T_{0}}, & \Delta^{\prime}=\frac{\Delta}{p(k / m) T_{0}}, \quad q^{\prime}=\frac{q}{p\left(\frac{k}{m} T_{0}\right)^{1 / 2}}, \\
x^{\prime} & =\frac{x}{a}, \quad \sigma^{\prime}=\frac{a T_{0}}{p\left(\frac{k}{m} T_{0}\right)^{1 / 2}} \sigma
\end{aligned}
$$

Reduced variables (10) are used elsewhere below, and we omit primes in order to save notation.

In terms of variables (10), Grad's equations (8) for the BGK model may be written,

$$
\begin{aligned}
\partial_{x}(\Delta+15 T) & =-6 \frac{1}{K_{\mathrm{BGK}}} q, \\
\partial_{x} T & =-\frac{1}{28} \frac{1}{q K_{\mathrm{BGK}}} \Delta,
\end{aligned}
$$

where

$$
K_{\mathrm{BGK}}=\left(\frac{k T_{0}}{m}\right)^{1 / 2} \frac{\tau}{a},
$$

is Knudsen number. The local entropy production (9) for the BGK model takes the form,

$$
\sigma=\frac{1}{K_{\mathrm{BGK}}}\left\{\frac{2}{5} \frac{q^{2}}{T^{2}}+\frac{1}{120} \frac{\Delta^{2}}{T^{3}}\right\} .
$$


Equations (11) are easily solved analytically to give

$$
\begin{aligned}
T(x) & =T_{0}+W(\exp (-x / s)-1)-\frac{2}{5} \frac{q x}{K_{\mathrm{BGK}}}, \\
\Delta(x) & =-15 W \exp (-x / s)+\frac{56}{5} q^{2}, \\
W & =\frac{T_{1}-T_{0}+2 q /\left(5 K_{\mathrm{BGK}}\right)}{\exp (-1 / s)-1}, \\
s & =-\frac{28}{15} q K_{\mathrm{BGK}}
\end{aligned}
$$

We assume $T_{1}>T_{0}$, then the meaningful values of the heat flux $q$ are negative. Exponential decay near the cold boundary $x=0$ indicates the boundary layer, and the absolute value of $s$ represents its effective thickness (note that if $q<0$ then $s>0$ ). Notice that this thickness depends both on the Knudsen number, and on the yet unknown boundary condition $\hat{q}$.

As it has been suggested in the previous section, we study minima of the one-parametric family of the layer-averaged entropy productions,

$$
\Sigma_{L}(q)=\frac{1}{2 L}\left(\int_{0}^{L} \sigma(x, q) d x+\int_{1-L}^{1} \sigma(x, q) d x\right) .
$$

For small Knudsen numbers, and small difference of wall temperatures, results can be compared with the analytical estimate for $q$ drawn from the conventional Fourier law. In that case, as it follows from the Chapman-Enskog solution [20], $q=-(5 / 2) K_{\mathrm{BGK}} \partial_{x} T$. This allows to analytically estimate the heat flux as $q^{*} \approx-(5 / 2) K_{\mathrm{BGK}}\left(T_{1}-T_{0}\right)$. In the test discussed below the following set of parameters has been used: $T(1)=1.1, T(0)=1$, and $K_{\mathrm{BGK}}=0.05$, which results in the analytical estimate, $q^{*}=-0.0125$ for the heat flux, and $|s| \approx 10^{-3}$ for the characteristic thickness of the boundary layer corresponding to this estimate.

First we compare functionals $\Sigma_{L}$ for various layer widths $L$. Fig. 1 demonstrates the layeraveraged entropy production $\Sigma_{L}(q)$ for different boundary layer thickness $L$, including the limit of the infinitely thin layer, $\lim _{L \rightarrow 0} \Sigma_{L}(q)$, as well as total entropy production, and the functional of Struchtrup and Weiss (4).

We observe that, when $L$ varies from 1 to 0 , there are two qualitatively different outcomes for the entropy production $\Sigma_{L}$. For $L$ larger than a crossover value $L_{\mathrm{c}}$, function $\Sigma_{L}(q)$ (14) has one 
unphysical minimum $q=0$, which coincides with the minimum of the total bulk-averaged entropy production (3). The latter unphysical minimum has been already reported by Struchtrup and Weiss [8]. However, for $L \leq L_{\mathrm{c}}$, function $\Sigma_{B_{L}}(q)$ (14) demonstrates another local minimum, $q_{\min }(L)$, although the unphysical minimum is still present. As it is seen from Fig. 1, variations of the value $q_{\min }(L)$ is small within the interval $\left[0, L_{\mathrm{c}}\right]$, and all the values $q_{\min }(L)$ are close to the analytical estimate $q^{*}$, on the one hand, and on the other hand, these values are close to the minimizer of the function (4). This happens because the maximum of the local entropy production in this and similar cases appears to be at the boundary, or within the boundary layer. It is also remarkable that there is invariant point where all curves $\Sigma_{L}(q)$ almost touch the curve corresponding to the total entropy production. This point is almost the same for any choice of $L$ and it is located very closely to the minimum of the function $\Psi_{1}(q)(6)$.

Fig. 2 compares the three potentials, $\Psi_{1}(6), \Psi_{2}(7)$, and $\Psi_{\mathrm{sw}}(4)$. The value of boundary width $L$ in the definition of potentials $\Psi_{1}$ and $\Psi_{2}$ was fixed with help of estimate $L(q)=s(q)(13)$ which is the function of boundary condition $q$. The minima of these functionals correspond to the following values of $q$ :

$$
\begin{array}{r}
q_{\min }\left[\Psi_{1}\right]=-0.012526 \\
q_{\min }\left[\Psi_{2}\right]=-0.012505 \\
q_{\min }\left[\Psi_{\mathrm{sw}}\right]=-0.012473
\end{array}
$$

All these values are very close to the analytical estimate $q^{*}=-0.012500$. Notice that the estimate $q^{*}$ corresponds to the most homogeneous profiles of the local entropy production, and also of the temperature (see Fig. 3, Fig. 4, and Fig. 5, 6). Namely, one observes that if the values $q$ are not in small vicinity of $q^{*}$ there is an active domain near left wall $x=0$ where an exponential decay shows up. It is interesting to note that the boundary layer near right boundary does not have any such activity, what is a consequence of the fact that at this boundary the temperature flux is directed outward the bulk. In spite of slight deviations in the results obtained with help of different potentials they give practically the same temperature profiles.

Although predictions based on all the three potentials, (6), (7), and (4), are close to each other 
in the case of small Knudsen number, we have noticed considerable divergency for larger Knudsen number. In order to address this point, we have increased the value of the parameter $K_{\mathrm{BGK}}$, but have lowered the value for the dimensionless temperature difference. Figures 7 and 8 correspond to the parameter set $K_{\mathrm{BGK}}=0.5$, and $T_{1}-T_{0}=0.01$. We then are able to qualitatively compare this result with the direct solutions to the linearized BGK equations reported in the Ref. [19]. There is a clear indication that when the temperature difference between the walls is sufficiently small the solution of BGK kinetic equations gives almost linear temperature profiles in the bulk even for large Knudsen numbers. Solution based on our variational principles confirms to this picture qualitatively. However Figures 9 and 10 indicate that the Struchtrup-Weiss functional $\Psi_{\text {sw }}$ point out the solution which is considerably far from "almost linear" unlike the case of small Knudsen numbers, which proves that our boundary layer functional are more relevant to the problem of selection of boundary conditions.

Similar analysis has been performed for the model of Maxwell molecules. In terms of variables (10), Grad's moment system for the MM model reads:

$$
\begin{aligned}
\partial_{x}(\Delta+15 T) & =-4 \frac{1}{K_{\mathrm{MM}}} \frac{q}{T}, \\
\partial_{x} T & =-\frac{1}{42} \frac{1}{K_{\mathrm{MM}}} \frac{\Delta}{q T},
\end{aligned}
$$

where Knudsen number $K_{\mathrm{MM}}$ is,

$$
K_{\mathrm{MM}}=\frac{\left(k T_{0} / m\right)^{3 / 2}}{\alpha p a} .
$$

The local entropy production takes the form,

$$
\sigma=\frac{1}{15 K_{\mathrm{MM}}}\left\{4 \frac{q^{2}}{T^{3}}+\frac{1}{12} \frac{\Delta^{2}}{T^{4}}\right\}
$$

Because of the nonlinearity, equations (16) were solved numerically. For small Knudsen numbers, and small difference of the wall temperatures, the heat flux has been estimated as $q^{*} \approx-(15 / 4) K_{\mathrm{MM}} T^{\prime}\left(T_{1}-T_{0}\right)$, where $T^{\prime}=\left(T_{0}+T_{1}\right) / 2$. With this, the boundary layer is estimated as $L \approx \frac{42}{15} K_{\mathrm{MM}} T^{\prime}|q|$. Like for BGK model we have input the latter estimation into the expressions (6) and (7) in order to completely specify the functions $\Psi_{1}(q)$ and $\Psi_{2}(q)$. In the test 
presented below the following parameters were used: $K_{\mathrm{MM}}=0.05, T_{0}=1.0$, and $T_{1}=1.1$, which results in the estimate $q^{*} \approx-0.01969$.

All results for the MM model are similar to those for the BGK model discussed above. Fig. 9 demonstrates the crossover in the structure of the layer-averaged entropy production under variation of the layer width. Potentials $\Psi_{1}, \Psi_{2}$ and $\Psi_{\mathrm{sw}}$ are compared in Fig. 10. Corresponding minima of these potentials occur at the following values of the heat flux:

$$
\begin{aligned}
q_{\min }\left[\Psi_{1}\right] & =-0.019777, \\
q_{\min }\left[\Psi_{2}\right] & =-0.019714, \\
q_{\min }\left[\Psi_{\mathrm{sw}}\right] & =-0.019643 .
\end{aligned}
$$

All these values agree well with the estimate $q^{*} \approx-0.01969$. Notice that in both the BGK and the MM models, potential $\Psi_{2}$ gives the result most close to the analytical prediction. Temperature and local entropy production profiles are demonstrated in Fig. 11 and Fig. 12.

\section{CONCLUSION AND DISCUSSION}

In this paper, we have studied possibilities of introducing a variational principle for boundary conditions for Grad moment equations. Our approach is based on a systematic introduction of the boundary layer into a phenomenology of variational principles. The approach has been tested for models of heat conduction suggested earlier. We have observed that variation of the thickness of the domain taken to represent the boundary layer results in a crossover: When $L>L_{\mathrm{c}}$ then the minimum of the layer-averaged entropy production corresponds to the one predicted be the total bulk-averaged entropy production. However, if $L<L_{\mathrm{c}}$, the second local minimum appears, and which corresponds to the estimate close to the one resulting from the Struchtrup-Weiss minimax principle. This crossover gives an opportunity to define the boundary layer without restoring to more precise but also more elaborative microscopic considerations. This observation has led us to variational principles which compare the average entropy production in the boundary layer and

in the bulk. The results have been found in excellent agreement with analytical predictions. The 
results of this study therefore make us confident in the usefulness of the entropy production in the boundary layer for the problem of boundary conditions in the extended thermodynamic systems. The approach is computationally more advantageous than the use of the minimax principle of [8] since it avoids a computationally intensive operation of finding extrema of this entropy production in entire volume, rather, it is based on a simple integral measure and allow to use simplifications for small boundary layer width.

Finally, it should be stressed that, while the problem of boundary conditions for moment equations (and, more broadly, for stationary thermodynamic systems with additional fields) can be addressed indeed through consideration of plausible minimum principles, the complete understanding of those can be accomplished only in the framework of dynamic approach to the boundary condition. This point is left for future work. 


\section{REFERENCES}

[1] R.B. Bird, C.F. Curtiss, R.C. Armstrong, and O Hassager, Dynamics of Polymeric Fluids (John Wiley and Sons, New York, 1987).

[2] H. Grad, Comm. Pure and Appl. Math. 2, 331 (1949).

[3] M. M. Veinberg, Variational Methods for the Study of Nonlinear Operators (Holden-Day, San Francisco, 1964).

[4] O. C. Zienkiewicz and K. Morgan, Finite Elements and Approximations (Wiley Interscience, New York, 1983).

[5] M. C. Gross and P. C. Hohenberg, Rev. Mod. Phys. 65, 851 (1993).

[6] M. Grmela, J. Non-Newtonian Fluid Mech. 96, 221 (2001).

[7] J. D. Shore, D. Ronis, L. Piché and M. Grant, Phys. Rev. Lett. 77, 655 (1996).

[8] H. Struchtrup and W. Weiss, Phys. Rev. Lett. 80, 5048 (1998).

[9] J. Au, I. Müller, and T. Ruggeri, Continuum Mech. Thermodyn. 12, 19 (2000).

[10] I. Prigogine, Introduction to Thermodynamics of Irreversible Processes (Interscience, New York, 1967).

[11] P. Glansdorff and I. Prigogine, Physica A 30, 351 (1964).

[12] A. M. Kogan, Prikl. Mat. Mech. 29, 122 (1965).

[13] I. Müller and T. Ruggeri, Extended Thermodynamics (Springer, New York, 1993).

[14] C. D. Levermore, J. Stat. Phys. 83, 1021 (1996).

[15] I. V. Karlin, Proc. VIII USSR Symp. on Burning and Combustion, (Tashkent, 1986), p. 97.

[16] I. V. Karlin, in: Mathematical Problems of Chemical Kinetics, Eds. K. Zamaraev and G. S. Yablonskii (Nauka, Novosibirsk, 1989). 
[17] A. N. Gorban and I. V. Karlin, Phys. Rev. E 54, 3109 (1996).

[18] C. Cercignani, The Boltzmann Equation and Its Applications, (Springer-Verlag, New York, 1988).

[19] P. Bassanini, C. Cercignani and C. D. Pagani, Int. J. Heat and Mass Transfer 10, 447 (1967).

[20] S. Chapman and T. Cowling, Mathematical Theory of Non-Uniform Gases (Cambridge University Press, Cambridge, 1958). 


\section{FIGURES}

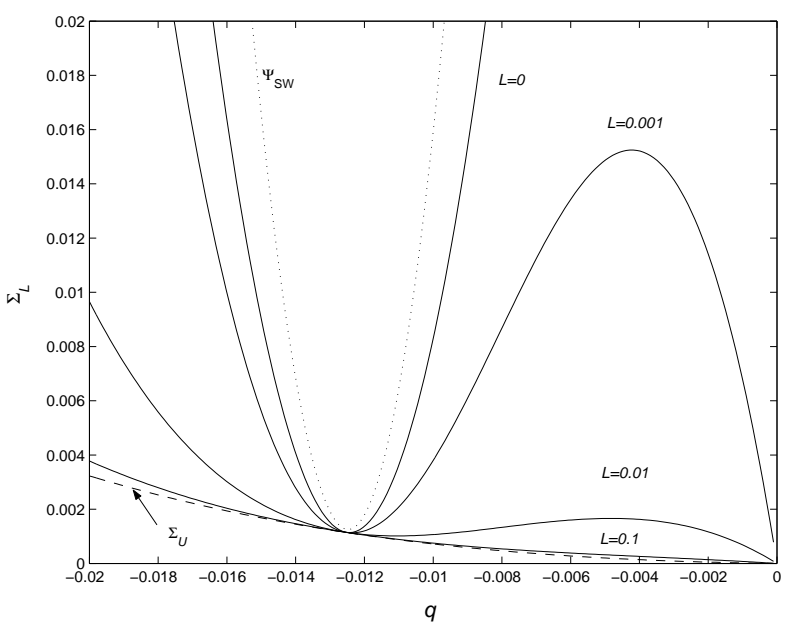

FIG. 1. Layer-averaged entropy production $\Sigma_{L}(q)(14)$ as a function of the boundary condition $q$ for different layer widths $L$ in the BGK model with $K_{\mathrm{BGK}}=0.05$ and $T_{1}-T_{0}=0.1$. Dashed line is the total bulk-averaged entropy production.

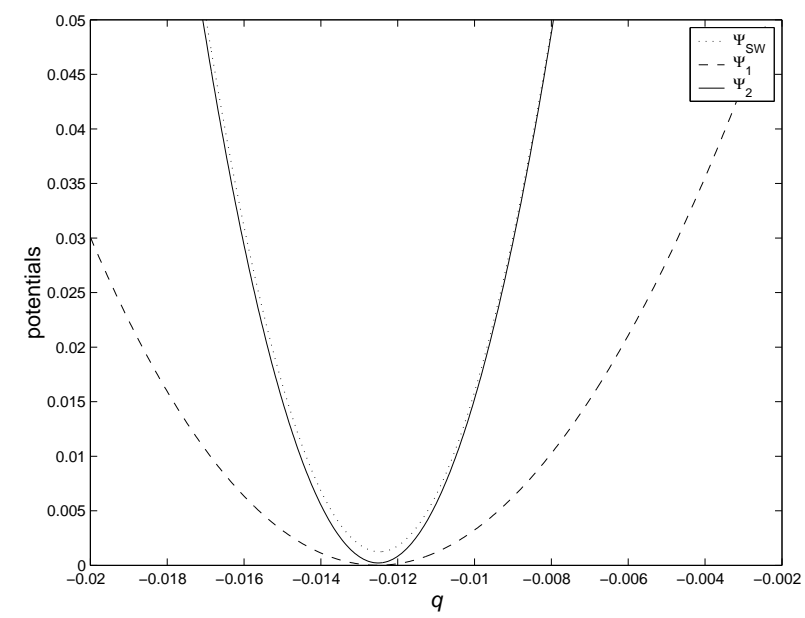

FIG. 2. Comparison of the potentials $\Psi_{1}\left(\right.$ (6) and $\Psi_{2}$ (7) with the Struchtrup-Weiss potential $\Psi_{S W}$ (4) in the BGK model with $K_{\mathrm{BGK}}=0.05$ and $T_{1}-T_{0}=0.1$. 


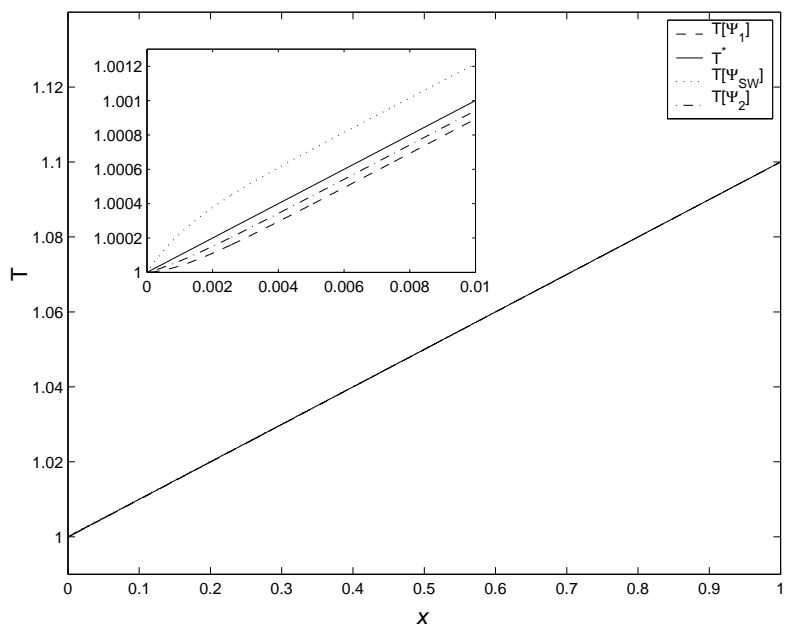

FIG. 3. Profiles of the reduced temperature $T$ in the BGK model with $K_{\mathrm{BGK}}=0.05$ and $T_{1}-T_{0}=0.1$ corresponding to optimization with various functionals.

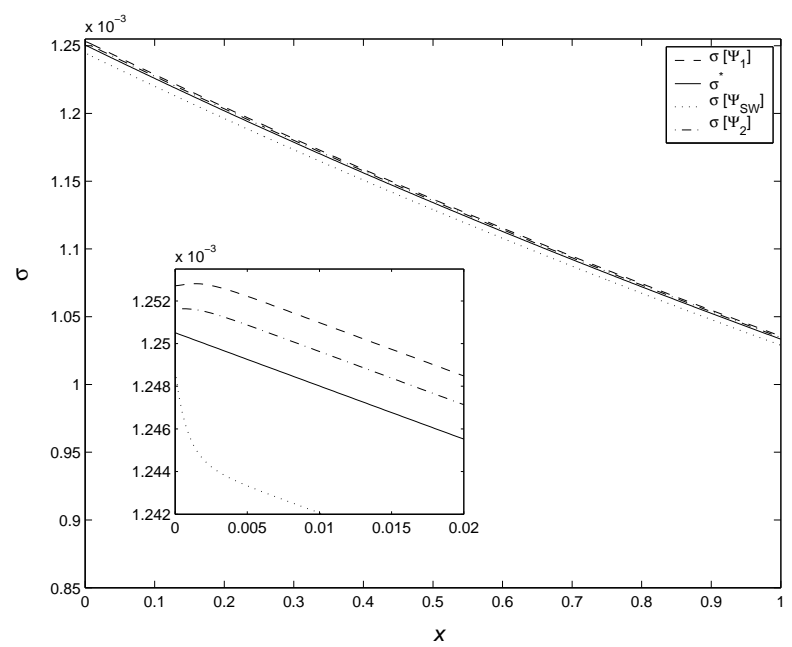

FIG. 4. Profiles of the reduced local entropy production $\sigma$ (b) in the BGK model with $K_{\mathrm{BGK}}=0.05$ and $T_{1}-T_{0}=0.1$ corresponding to optimization with various functionals. 


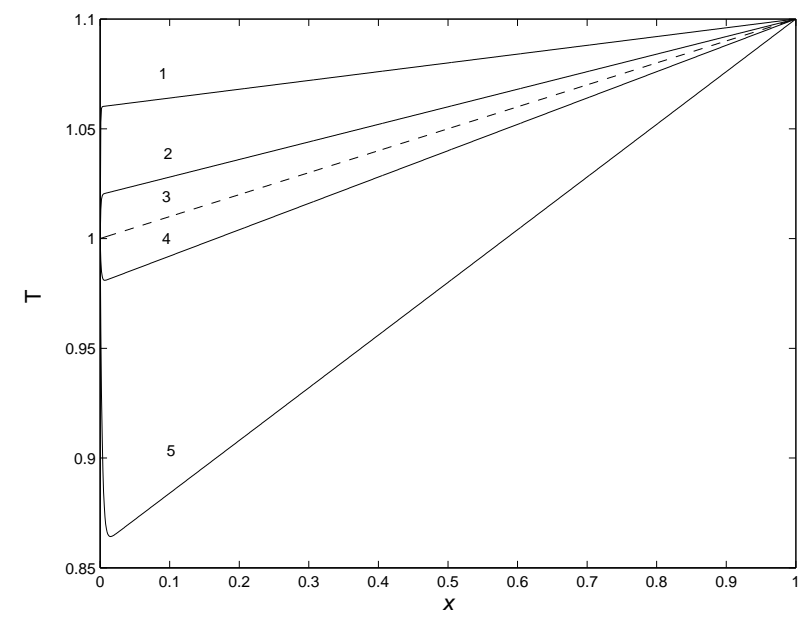

FIG. 5. The reduced temperature $T$ in BGK model with $K_{\mathrm{BGK}}=0.05$ and $T_{1}-T_{0}=0.1$ for large deviations of boundary condition $q$ from its optimal value $q^{*}=-0.0125$ : curve 1 corresponds to $q=-0.005$, curve 2 to $q=-0.01$, curve 3 to $q=q^{*}$ (Fourier law), curve 4 to $q=-0.015$, curve 5 to $q=-0.03$.

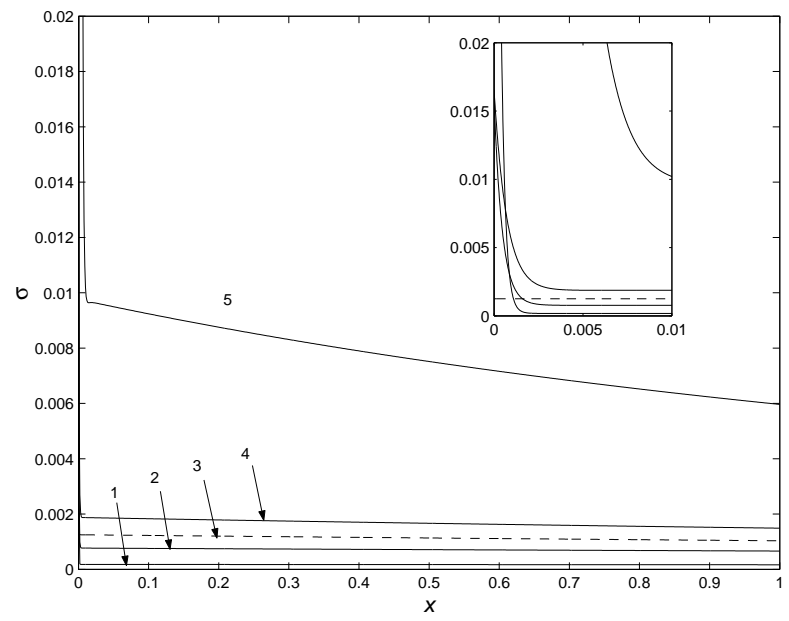

FIG. 6. The reduced reduced local entropy production $\sigma$ in BGK model with $K_{\mathrm{BGK}}=0.05$ and $T_{1}-T_{0}=0.1$ for large deviations of boundary condition $q$ from its optimal value $q^{*}=-0.0125$ : curve 1 corresponds to $q=-0.005$, curve 2 to $q=-0.01$, curve 3 to $q=q^{*}$ (Fourier law), curve 4 to $q=-0.015$, curve 5 to $q=-0.03$. 


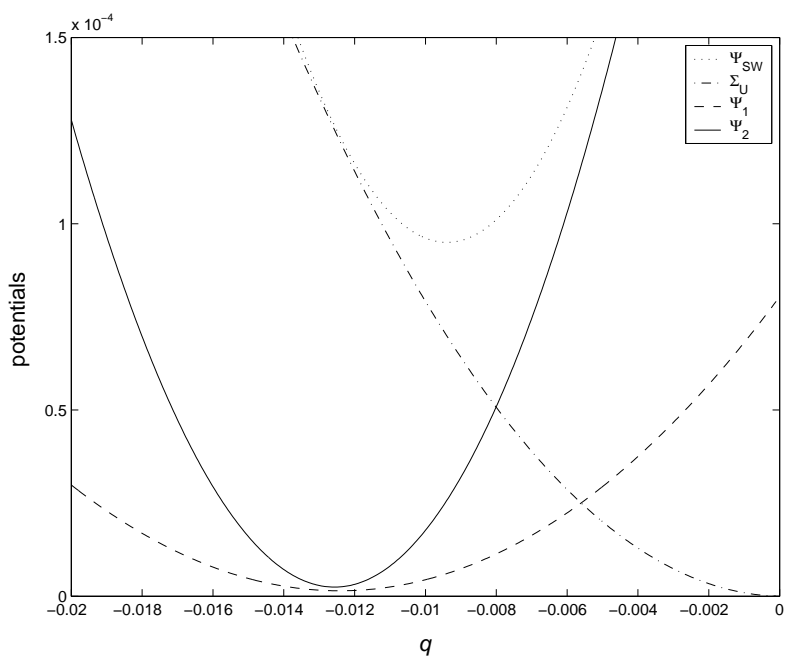

FIG. 7. Functionals $\Psi_{1}(6)$ and $\Psi_{2}\left(\right.$ (7) as compared to Struchtrup-Weiss potential $\Psi_{\mathrm{sw}}$ for the BGK model with moderate Knudsen number, $K_{B G K}=0.5\left(T_{1}-T_{0}=0.01\right)$.

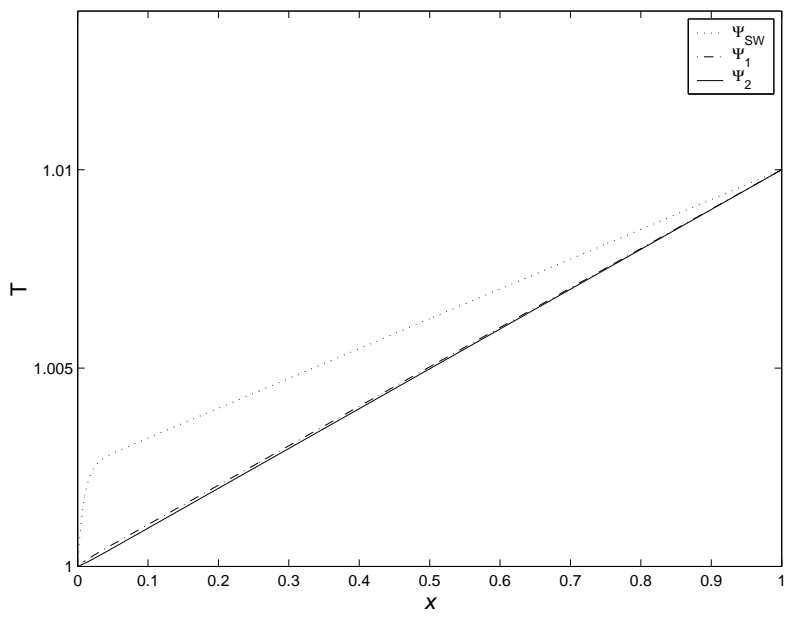

FIG. 8. Temperature profiles for moderate Knudsen number, $K_{\mathrm{BGK}}=0.5\left(T_{1}-T_{0}=0.01\right)$, in the BGK model. 


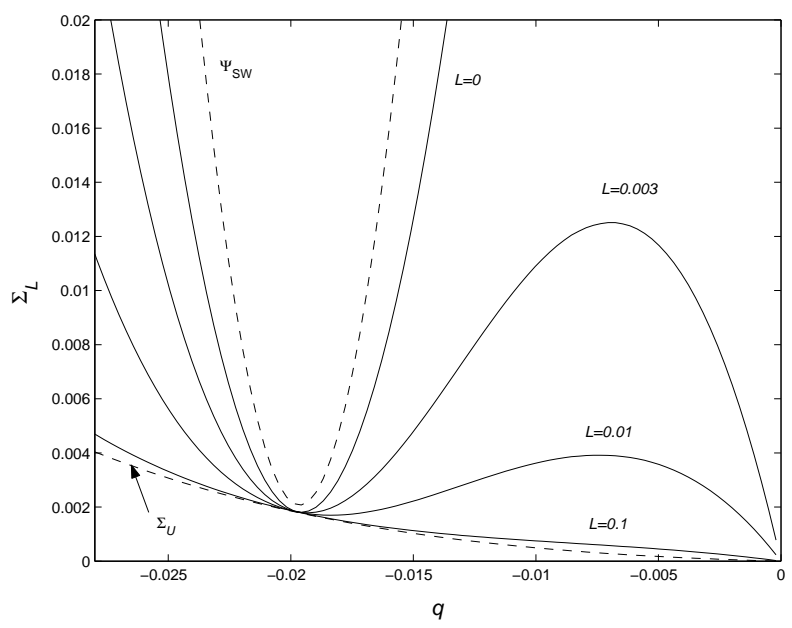

FIG. 9. Layer-averaged entropy production $\Sigma_{L}(q)(14)$ as a function of the boundary condition $q$ for different layer widths $L$ in the Maxwell molecules model $\left(K_{\mathrm{MM}}=0.05\right)$. Dashed line is the total bulk-averaged entropy production.

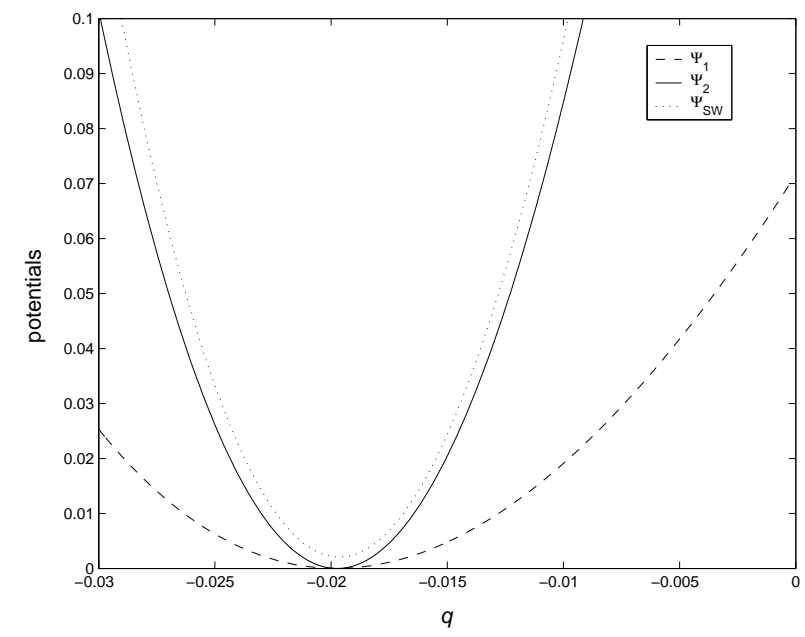

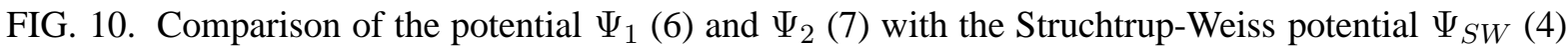
for the model of Maxwell molecules. 


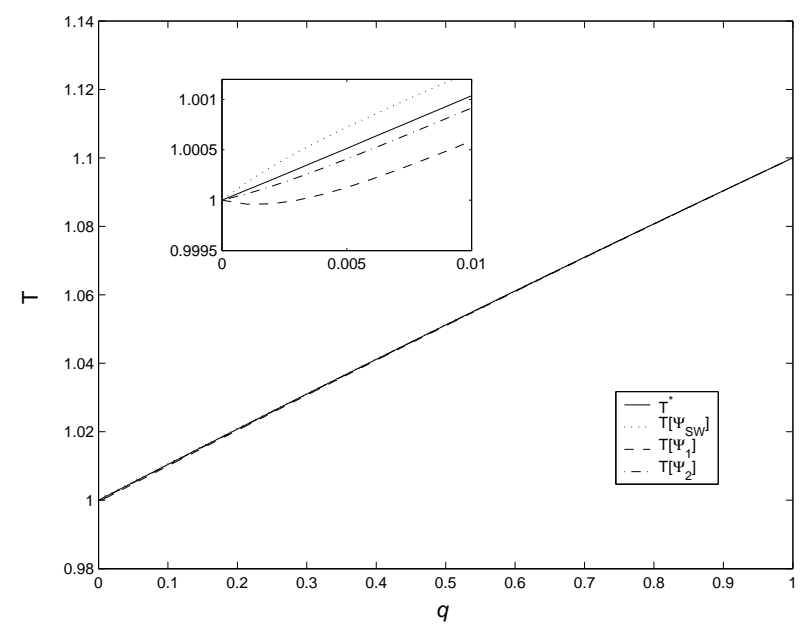

FIG. 11. Profiles of the reduced temperature $T$ in the model of Maxwell molecules corresponding to minima of various potentials.

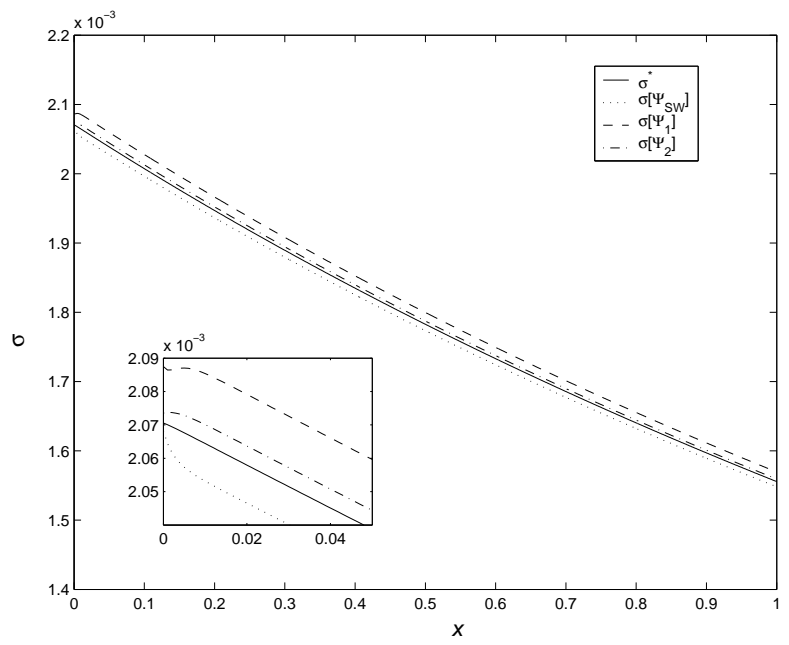

FIG. 12. Profiles of the reduced local entropy production $\sigma$ in the model of Maxwell molecules corresponding to minima of various potentials. 